\title{
Studies on Approximation Methods in Calculating the Magnetic Dipolar Interaction Energy, and Its Impact on the Relaxation Time of Magnetic Nanoparticle Systems
}

\author{
M. CACCIOLA ${ }^{a, *}$ AND A. BERDIE ${ }^{b}$
}

${ }^{a}$ University "Mediterranea" of Reggio Calabria, DICEAM, Via Graziella Feo di Vito, I-89100 Reggio Calabria, Italy

b"Politehnica" University of Timisoara, Department of Electrical Engineering and Industrial Informatics,

Piata Victoriei No. 2, 300006 Timisoara, jud. Timis, Romania

(Received May 4, 2015; in final form November 25, 2015)

\begin{abstract}
The studies on monodomain magnetic nanoparticle systems in colloidal suspensions have heightened lately due to their technological applications, in particular in medicine. Most applications depend on the behaviour of these systems in external magnetic field. In these systems, the nanoparticle dynamics are characterized by the Néel relaxation time and Brownian relaxation time. Due to the complexity of these systems, modelling and numerical simulation, requiring some methods of calculation, are used in the studies. Lately, it has been experimentally and theoretically shown that the magnetic dipolar interactions among nanoparticles influence the behaviour of the systems, even at low concentrations of nanoparticles. The complexity of the problem related to this type of interaction comes from its long-range anisotropic characteristic. This paper presents a series of studies on how the approximation methods, used for the dipolar magnetic interaction energy calculation, affect the magnetic nanoparticle relaxation time, as well as the impact of this aspect on the interpretation of results.
\end{abstract}

DOI: $10.12693 /$ APhysPolA.129.88

PACS: $75.75 . \mathrm{Fk}$

\section{Introduction}

The magnetic nanoparticles (MNPs) are parts of magnetic materials with typical nanometrical size (usually $<100 \mathrm{~nm}$ ). They are naturally found in various materials, but can be also artificially made and used as the component of nanofluids, biomedical materials, permanent magnets, soft magnetic materials, etc. Their properties are very interesting, both from a scientific and a technological point of view. Some of the most important applications of the nanoparticles are: biomedical application, high density magnetic data storage media, magneto-optical switches, sensors based on giant magnetoresistance, etc.

The studies on monodomain MNPs systems in colloidal suspensions are recently becoming more and more numerous, since their use in medical applications such as: magnetic hyperthermia, magnetic resonance imaging, drug targeting, biomarkers, biosensors, and separation of biomolecules [1-3]. The applications in medical diagnostics and therapy require the magnetic nanoparticles be stable in water at neutral $\mathrm{pH}$ and physiological salinity. The system obtained under these conditions represents a magnetic nanofluid system. The most commonly used nanoparticles in biomedical applications are the iron-oxide nanoparticles $\mathrm{Fe}_{3} \mathrm{O}_{4}$ and $\gamma-\mathrm{Fe}_{2} \mathrm{O}_{3}$ [1]. The volume fraction of magnetic materials has usually just few percent.

The simulation and modelling are successfully used in science and engineering [1-8]. Regarding the models which describe the magnetic behaviour of nanopar-

*corresponding author; e-mail: matteo.cacciola@unirc.it ticle systems, the literature presents theoretical models and simulation models. Some theoretical models, such as Dormann-Bessais Fiorani and Morup-Tronc [9], are generally applicable for weak interactions and use a series of approximations, more or less justified. However, they have the advantage to provide analytical expressions for extracting the system parameters. The simulation models offer an efficient simulation scheme and have the major advantage to rigorously treat the local and temporal fluctuations of the macroscopic quantities characterizing the system. The mostly used simulation methods are Monte Carlo [10-12] and the magnetization dynamics [4]. Between theoretical and simulation models, we can set the so-called stochastic-phenomenological models [13-16].

Regardless of the model adopted, a method to calculate the dipolar magnetic energy of the system and the local field on the nanoparticle is highly recommended. In fact, the role of dipolar interactions is really important in a number of applications, but still not well understood [17]. In this work, we propose a study about suitable modelling the calculation of the magnetic dipolar field or energy, with some discussion about the relationships between the simulation performances and the modelling parameters.

\section{Long-range magnetic dipole interactions and} calculation methods

Being the nanoparticles monodomainal, the quantummechanical exchange interaction between the electronic spins can keep all the atomic moments aligned. Therefore, the total magnetic moment $\mu_{i}$ of the nanoparticle has the order of about $10^{4} \mu_{B}$ [18]. Thus, each nanoparticle is characterized by a magnetic moment, which is usually given by [19]:

$$
\boldsymbol{\mu}_{i}=M_{s} v_{i} \hat{\mu}_{i}
$$


where $M_{s}$ is the spontaneous magnetization, $v_{i}$ is the particle volume and $\hat{\mu}_{i}$ is the unit vector of the magnetic moments.

Let $\boldsymbol{\mu}_{i}$ be the magnetic moment of the nanoparticle $i$-th, and $\boldsymbol{\mu}_{j}$ the magnetic moment of the $j$-th nanoparticle with uniaxial anisotropy (Fig. 1). The energy of the dipolar magnetic interaction between two nanoparticles $(i$ and $j$ ) is given by the equation

$$
E_{i j d}=\frac{\mu_{0}}{4 \pi} \frac{\mu_{i} \mu_{j}}{r_{i j}^{3}}\left[\hat{\mu}_{i} \cdot \hat{\mu}_{j}-3\left(\hat{\mu}_{i} \cdot \hat{r}_{i j}\right)\left(\hat{\mu}_{j} \cdot \hat{r}_{i j}\right)\right]
$$

where $r_{i j}$ is the distance between the centres of those two nanoparticles, $\hat{r}_{i j}$ is the versor of the direction that connects the nanoparticles $i$ and $j, \hat{\mu}_{i}$ and $\hat{\mu}_{j}$ are the unit vectors of the magnetic moments of the nanoparticles $i$ and $j$, and $\mu_{0}$ is the magnetic permeability of vacuum (Fig. 1)

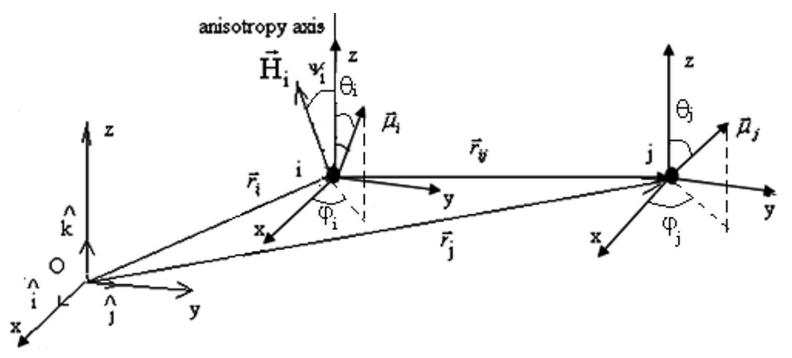

Fig. 1. Notation for the dipolar magnetic energy formula.

Equation (2) can be written as follows:

with

$$
E_{i j d}=-\mu_{0} \boldsymbol{\mu}_{i} \cdot \boldsymbol{H}_{i j d}
$$

$$
\boldsymbol{H}_{i j d}=\frac{\mu_{j}}{4 \pi r_{i j}^{3}}\left[3 \hat{r}_{i j} \cdot\left(\hat{\mu}_{j} \cdot \hat{r}_{i j}\right)-\hat{\mu}_{j}\right] .
$$

$\boldsymbol{H}_{i j d}$ is called local dipolar field due to the dipole-dipole magnetostatic interaction of the two nanoparticles. Since the nanoparticle $i$ has a dipole-dipole magnetostatic interaction with all the other nanoparticles, the dipolar magnetic energy of the nanoparticle $i$ and the local dipolar magnetic field acting on the nanoparticle $i$ can be expressed as follows:

$$
\begin{aligned}
E_{i d} & =\mu_{0} \sum_{j, j \neq i} \boldsymbol{\mu}_{i} \cdot \hat{H}_{i j d} \\
\boldsymbol{H}_{i d} & =\frac{1}{4 \pi} \sum_{j, j \neq i} \frac{\mu_{i}}{r_{i j}^{3}}\left[3 \hat{r}_{i j} \cdot\left(\hat{\mu}_{j} \cdot \hat{r}_{i j}\right)-\hat{\mu}_{j}\right] .
\end{aligned}
$$

Hereinafter, we are going to discuss several methods for the dipolar magnetic energy approximate calculation, in order to numerically assess the provided results. Direct summation in Eqs. (5) and (6) is also named analytical (or "brute force") method.

\section{1. "Approximation 1": the averaged method}

In some scientific papers, the configuration of randomly distributed nanoparticles, having magnetic moments and anisotropy axes aligned along the external magnetic field, is analyzed. In this case, the energy of the dipolar magnetic interaction between two nanoparticles $(i$ and $j$ ) is given by the first term of Eq. (20) [20]:

$$
E_{i j d} \approx \frac{\mu_{0}}{4 \pi} \frac{\mu_{i} \mu_{j}}{r_{i j}^{3}}
$$

Given Eq. (6), the local dipolar field can be expressed as

$$
\boldsymbol{H}_{i j d} \approx-\frac{\boldsymbol{\mu}_{j}}{4 \pi r_{i j}^{3}}
$$

In this approximation called "Approximation 1", the dipolar magnetic energy of the nanoparticle $i$ and the local dipolar field acting on the nanoparticle $i$ are

$$
\begin{aligned}
& E_{i d} \approx \frac{\mu_{0} \mu_{i}}{4 \pi} \sum_{j, j \neq i} \frac{\mu_{j}}{r_{i j}^{3}}, \\
& \boldsymbol{H}_{i d} \approx-\frac{1}{4 \pi} \sum_{j, j \neq i} \frac{\boldsymbol{\mu}_{j}}{r_{i j}^{3}},
\end{aligned}
$$

where $N$ is the number of nanoparticles in the system. This approximation is valid only in intense external magnetic fields, so that the axes of anisotropy and magnetic moments are aligned with the external magnetic field. On the contrary, very intense magnetic fields are not allowed in biomedical applications, because of interactions with organs and organic tissues [21]. Therefore, the range of values is usually between $11 \mathrm{kA} / \mathrm{m}$ to $30 \mathrm{kA} / \mathrm{m}$.

\section{2. "Approximation 2": the parallel particle-to-particle method}

Now, let us assume that $[22,23]$ :

1. adjacent magnetic moments of the nanoparticles are parallel;

2. the difference between the angles made by the magnetic moments of the nanoparticles with the direction connecting them is negligible;

3. the anisotropy axes are aligned with the external magnetic field.

In this situation, we obtain the approximation

$$
E_{i j d} \approx \frac{\mu_{0}}{4 \pi} \frac{\left(1-3 \cos ^{2} \theta_{i j}\right)}{r_{i j}^{3}} \mu_{i} \mu_{j},
$$

where $\theta_{i j}$ is the angle between $\boldsymbol{r}_{i j}$ and the direction of magnetic moments. Because of Eqs. (3) and (4),

$$
\boldsymbol{H}_{i j d} \approx-\frac{1}{4 \pi} \frac{\left(1-3 \cos ^{2} \theta_{i j}\right)}{r_{i j}^{3}} \boldsymbol{\mu}_{j} .
$$

In our study, we call this approximation as "Approximation 2". In this case, the dipolar magnetic energy of the nanoparticle $i$ and the local dipolar field acting on the nanoparticle $i$ are

$$
\begin{aligned}
& E_{i d} \approx \frac{\mu_{0} \mu_{i}}{4 \pi} \sum_{j, j \neq i} \frac{\left(1-3 \cos ^{2} \theta_{i j}\right)}{r_{i j}^{3}} \mu_{j} \\
& \boldsymbol{H}_{i d} \approx-\frac{1}{4 \pi} \cdot \sum_{j, j \neq i} \frac{\left(1-3 \cos ^{2} \theta_{i j}\right)}{r_{i j}^{3}} \boldsymbol{\mu}_{j} .
\end{aligned}
$$

Please note that this kind of approximation assumes aligned magnetic anisotropy axes of the nanoparticles. It 
is an unrealistic approximation from a geometrical point of view, since it is difficult to make configuration specified in point 1 of the previous list for real-world cases-ofstudy.

\section{3. "Approximation 3":}

the Dormann-Bessais-Fiorani method

Another approximation used in the theoretical models is the so-called Dormann-Bessais-Fiorani (DBF) approximation [9], which we call "Approximation 3" in this study. In this approximation, the anisotropy easy axis is considered to be parallel with the $z$ axis (Fig. 1). Let $\hat{u}_{x i j}, \hat{u}_{y i j}$ and $\hat{u}_{z i j}$ be the components of the vector found in the right bracket of Eq. (4), and $\hat{x}_{i j}, \hat{y}_{i j}$ and $\hat{z}_{i j}$, the components of the unit vector, $\hat{r}_{i} j$, with

$$
\boldsymbol{x}_{i j}^{2}+\hat{y}_{i j}^{2}+\hat{z}_{i j}^{2}=1 \text {. }
$$

Then, referring to Fig. 1:

$$
\begin{aligned}
& \hat{u}_{x i j}=\left(3 \hat{x}_{i j}^{2}-1\right) \sin \theta_{j} \cos \varphi_{j}+3 \hat{x}_{i j} \hat{y}_{i j} \sin \theta_{j} \sin \varphi_{j} \\
& \quad+3 \hat{x}_{i j} \hat{z}_{i j} \cos \theta_{j} \\
& \hat{u}_{y i j}=3 \hat{x}_{i j} \hat{y}_{i j} \sin \theta_{j} \cos \varphi_{j}+\left(3 \hat{y}_{i j}^{2}-1\right) \sin \theta_{j} \sin \varphi_{j} \\
& \quad+3 \hat{y}_{i j} \hat{z}_{i j} \cos \theta_{j} \\
& \hat{u}_{z i j}=3 \hat{x}_{i j} \hat{z}_{i j} \sin \theta_{j} \cos \varphi_{j}+3 \hat{y}_{i j} \hat{z}_{i j} \sin \theta_{j} \sin \varphi_{j} \\
& \quad+\left(3 \hat{z}_{i j}^{2}-1\right) \cos \theta_{j} .
\end{aligned}
$$

To simplify the expression of the dipolar magnetic energy $[24,25]$, DBF considered that the probabilities to find the magnetic moment of the $j$ nanoparticle at $\left(\theta_{j}, \varphi_{j}\right)$ and $\left(\theta_{j}, \varphi_{j}+\pi\right)$ are equal. Therefore, we can substitute $H_{i j d}$ with $H_{i j d}\left(\theta_{j}\right)=\frac{1}{2}\left[H_{i j d}\left(\theta_{j}, \varphi_{j}\right)+H_{i j d}\left(\theta_{j}, \varphi_{j}+\pi\right)\right]$. It is equivalent with the mediation of $\varphi_{j}$. By making this mediation in the components given by Eqs. (16)-(18), we obtain

$$
\boldsymbol{H}_{i j d} \approx \frac{1}{4 \pi r_{i j}^{3}} \boldsymbol{v}_{i j} \cos \theta_{j},
$$

where $\boldsymbol{v}_{i j}$ is a vector with the components $\left(3 \hat{x}_{i j} \hat{z}_{i j}, 3 \hat{y}_{i j} \hat{z}_{i j}, 3 \hat{z}_{i j}^{2}-1\right)$. With Eqs. (19), and (4), the expression of the dipolar magnetic energy becomes

$$
E_{i j d} \cong-\frac{\mu_{0} \mu_{i} \mu_{j}}{4 \pi r_{i j}^{3}} P\left(x_{i j}, y_{i j}, z_{i j}, \theta_{i}, \varphi_{i}\right) \cos \theta_{j}
$$

with $P(\boldsymbol{\alpha}, \boldsymbol{\beta}, \boldsymbol{\gamma}, \theta, \varphi)=3 \hat{\alpha} \hat{\gamma} \sin \theta \cos \varphi+3 \hat{\beta} \hat{\gamma} \sin \theta \sin \varphi+$ $\left(3 \hat{\gamma}^{2}-1\right) \cos \theta$. By averaging again on $\varphi_{i}$, i.e. by considering that the probabilities to find the magnetic moment of the nanoparticle $i$ at $\left(\theta_{j}, \varphi_{j}\right)$ and $\left(\theta_{j}, \varphi_{j}+\pi\right)$ are equal, we obtain

$$
E_{i j d} \cong-\frac{\mu_{0} \mu_{i} \mu_{j}}{4 \pi r_{i j}^{3}}\left(3 \hat{z}_{i j}^{2}-1\right) \cos \theta_{i} \cos \theta_{j} .
$$

Then, the dipolar magnetic energy of the nanoparticle $i$ and the local dipolar field act on the $i$-th particle (which has components only along the $z$ axis) are

$$
\boldsymbol{H}_{i d} \approx \boldsymbol{k} \frac{1}{4 \pi} \sum_{j, j \neq i} \frac{\mu_{j}\left(3 \hat{z}_{i j}^{2}-1\right)}{r_{i j}^{3}} \cos \theta_{j} .
$$

With this notation, we obtain

$$
E_{i d} \approx-\mu_{0} \mu_{i} H_{i d} \cos \theta_{i} .
$$

Since this approximation considers the case of aligned magnetic anisotropy axes of the nanoparticles, it is an unrealistic simplification of magnetic dipolar energy.

\subsection{The Ewald summation method}

The Ewald summation method, named after Paul Peter Ewald, is a method for computing long-range interaction in periodic systems. It was firstly developed as the method for calculating electrostatic energies of ionic crystals, and now it is commonly used for calculating longrange interactions in computational condensed matter. The Ewald summation replaces the summation of interaction energies in real space with an equivalent summation in Fourier space. In this method, the long-range interaction is divided into two parts: a short-range contribution, and a long-range contribution, which does not have singularity. The short-range contribution is calculated in a real-space, whereas the long-range contribution is calculated using a Fourier transform. The advantage of the Ewald summation is a rapid convergence of the energy compared with that of a direct summation. It means that the method has high accuracy and reasonable speed to compute long-range interactions. Moreover, this method does not involve simplifications regarding the geometry of the magnetic moments. The total paired potential energy of dipole-dipole interaction in a three dimensional periodic system is given by the following equation [26]:

$$
\begin{aligned}
E_{d} & =\frac{\mu_{0}}{4 \pi} \cdot \frac{1}{2} \sum_{i=1}^{N} \sum_{j=1}^{N} \sum_{n \in Z^{3}}\left\{\frac{\boldsymbol{\mu}_{i} \cdot \boldsymbol{\mu}_{j}}{\left|\boldsymbol{r}_{i j}+\boldsymbol{n}\right|^{3}}\right. \\
& \left.-\frac{3\left[\boldsymbol{\mu}_{i} \cdot\left(\boldsymbol{r}_{i j}+\boldsymbol{n}\right)\right] \cdot\left[\boldsymbol{\mu}_{j} \cdot\left(\boldsymbol{r}_{i j}+\boldsymbol{n}\right)\right]}{\left|\boldsymbol{r}_{i j}+\boldsymbol{n}\right|^{5}}\right\}
\end{aligned}
$$

where $\boldsymbol{r}_{i j}=\boldsymbol{r}_{i}-\boldsymbol{r}_{j}$. The sum over $\boldsymbol{n}$ is over all the simple cubic lattice points, $\boldsymbol{n}=\left(n_{x} L, n_{y} L, n_{z} L\right)$, with $n_{x}, n_{y}$, $n_{z}$, integers. The prime indicates that the $i=j$ term must be omitted for $\boldsymbol{n}=0$. The Ewald method provides an efficient way to calculate $E_{d}$, by splitting the problem into two convergent parts, one in real space and one in reciprocal space. The local magnetic field acting on the $i$-th nanoparticle is [26]:

$$
\boldsymbol{H}_{i d} \cong-\frac{1}{\mu_{0}} \frac{\partial E_{d}}{\partial \boldsymbol{\mu}_{i}} .
$$

The details of the method are discussed in the literature, some of the related references being [26-28]. This method has been widely applied in a number of papers, such as [29-31].

\section{Energy barriers and magnetic relaxation time}

In $3 \mathrm{D}$, in general, the energy of the $i$-th nanoparticle of the system in the local magnetic field $\boldsymbol{H}_{i}=\boldsymbol{H}_{i d}+\boldsymbol{H}$ is $[14-16]$ :

$$
E_{i}=-\mu_{0} \mu_{i} H_{i}\left(\hat{\mu}_{i} \cdot \hat{H}_{i}\right)-K_{i, \mathrm{eff}} V_{i}\left(\hat{\mu}_{i} \cdot \hat{e}_{i}\right)^{2}
$$

where $\hat{\mu}_{i}$ is the unit vector in the direction of magnetic moment of the $i$-th nanoparticle, $\hat{H}_{i}$ is the unit vector 
in the direction of local magnetic field acting on the $i$-th nanoparticle, $\hat{e}_{i}$ is the unit vector of the easy anisotropy axis of the $i$-th nanoparticle and $K_{i \text {,eff }}$ is the effective anisotropy constant of the $i$-th nanoparticle. In Ref. [32] it is supposed (without significant loss of generality) that the $\boldsymbol{H}_{i}$ acts on the plane $x O z$. In this case, Eq. (26) in spherical coordinates is

$$
\begin{aligned}
& E_{i}\left(\theta_{i}, \varphi_{i}\right)=-K_{i, \mathrm{eff}} V_{i} \cos ^{2} \theta_{i}-\mu_{0} \mu_{i} H_{i}\left(\cos \theta_{i} \cos \Psi_{i}\right. \\
& \left.\quad+\sin \theta_{i} \sin \Psi_{i} \cos \varphi_{i}\right) .
\end{aligned}
$$

The polar axis is the easy axis of the $i$-th nanoparticle. $\Psi_{i}$ is the angle between $\boldsymbol{H}_{i}$ and the easy axis of the $i$-th nanoparticle, and finally $0 \leq \theta_{i} \leq \pi, 0 \leq \varphi_{i} \leq 2 \pi$ are the elevation and azimuth angles of the $i$-th particle in polar coordinates, respectively.

The stationary points of the energy occur for $\varphi_{i}=0$ and $\varphi_{i}=\pi$. The stationary point for $\varphi_{i}=\pi$ corresponds to a maximum of $E_{i}\left(\theta_{i}, \varphi_{i}\right)$ in Eq. (27), and so is not of our interest. The stationary points at $\varphi_{i}=0$, however, correspond to a saddle point of $E_{i}\left(\theta_{i}, \varphi_{i}\right)$.

Under these assumptions, we applied the two state model that provides analytical solutions for barriers at $\Psi_{i}=0$ and $\Psi_{i}=\pi / 2$. For other values of $\Psi_{i}$, the solutions are approximated according to Pfeiffer [33], taking into account the random anisotropy axes of the particles.

In case of $\Psi_{i}=0$ and weak local magnetic field (e.g. in medical applications), the magnetic moment of a given nanoparticle $i$ can be in one of the two equilibrium states, with minimum energies determined by $\theta_{i}=0$ or $\theta_{i}=\pi$. $\theta_{i}$ is the angle between the easy axis and the $i$-th magnetic moment. These minima are separated by the maximum. The energy barriers for these re-orientations are

$$
\begin{aligned}
& E_{\mathrm{b} 12}^{i}=K_{i, \mathrm{eff}} V_{i}\left[1+\frac{\mu_{0} M_{s} H_{i}}{2 K_{i, \mathrm{eff}}}\right]^{2}, \\
& E_{\mathrm{b} 21}^{i}=K_{i, \mathrm{eff}} V_{i}\left[1-\frac{\mu_{0} M_{s} H_{i}}{2 K_{i, \mathrm{eff}}}\right]^{2} .
\end{aligned}
$$

When the $i$-th magnetic moment is normally oriented on $\boldsymbol{H}_{i}, \Psi_{i}=\pi / 2$. In this case,

$$
E_{\mathrm{b} 12}^{i}=E_{\mathrm{b} 21}^{i}=K_{i, \mathrm{eff}} V_{i}\left[1-\frac{\mu_{0} M_{s} H_{i}}{2 K_{i, \mathrm{eff}}}\right]^{2} .
$$

For other $\Psi_{i}$ values, in weak local magnetic field, we can apply the Pfeiffer approximation [33] and the lower energy barrier in accordance with this approximation

$$
\begin{aligned}
& E_{\mathrm{b}}^{i}=K_{i, \mathrm{eff}} V_{i}\left[1-\frac{\mu_{0} M_{s} H_{i}}{2 K_{i, \mathrm{eff}} g\left(\Psi_{i}\right)}\right]^{\alpha\left(\Psi_{i}\right)}, \\
& E_{\mathrm{b} 12}^{i}=E_{\mathrm{b} 21}^{i}=E_{\mathrm{b}}^{i},
\end{aligned}
$$

where

$$
\begin{aligned}
& g\left(\Psi_{i}\right)=\left(\cos ^{2 / 3}\left(\Psi_{i}\right)+\sin ^{2 / 3}\left(\Psi_{i}\right)\right)^{-3 / 2}, \\
& \alpha\left(\Psi_{i}\right)=0.86+1.14 g\left(\Psi_{i}\right) .
\end{aligned}
$$

Please, we note $H_{i}$ as the norm of $\boldsymbol{H}_{i}$ in Eqs. (28)-(31).

In order to find the reversal probability, we use a stochastic model. It assumes that the rotations of magnetic moments due to the thermal fluctuations can be modelled through the equation of probability. In this way, it is possible to find the magnetic moments of the particles at the time $t$, in the equilibrium state (1 or 2$)$. Let $P_{1 i}(t)$ and $P_{2 i}(t)$ be the probabilities to find the energy of the $i$-th particle into the state 1 or 2 at the time $t$, respectively. With $\nu_{12}^{i}$ and $\nu_{21}^{i}$, we denote the probabilities of transition, in time unit, from the state 1 to the state 2 and vice versa, respectively. With these notations, we obtain $[34,35]$ :

$$
\frac{\mathrm{d} P_{1 i}}{\mathrm{~d} t}=\nu_{21}^{i} P_{21}(t)-\nu_{12}^{i} P_{12}(t)
$$

provided the normalisation condition

$$
\begin{aligned}
& P_{12}(t)+P_{21}(t)=1, \\
& \nu_{k l}^{i}=\frac{1}{\tau_{k l}^{i}}=\frac{1}{\tau_{0}} \exp \left(-\frac{E_{\mathrm{b} k l}^{i}}{k_{\mathrm{B}} T}\right), k=1,2 ; l=2,1
\end{aligned}
$$

where $f_{0}=1 / \tau_{0}$ is the attempt frequency of magnetic reversal, usually considered having a constant value $f_{0}=10^{9} s^{-1}$. Thus, the Néel relaxation time $\tau_{\mathrm{N}}^{i}$ for the particle $i$ is given by

$$
\begin{aligned}
& \frac{1}{\tau_{\mathrm{N}}^{i}}=\frac{1}{\tau_{12}^{i}}+\frac{1}{\tau_{21}^{i}}=\frac{1}{\tau_{0}}\left[\exp \left(-\frac{E_{\mathrm{b} 21}^{i}}{k_{\mathrm{B}} T}\right)\right. \\
& \left.\quad+\exp \left(-\frac{E_{\mathrm{b} 12}^{i}}{k_{\mathrm{B}} T}\right)\right] .
\end{aligned}
$$

In the magnetic nanofluid, besides the Néel relaxation processes, there are Brownian relaxation processes. These relaxation processes are due to physical rotation of the nanoparticle in the base liquid. The Brownian relaxation time is given by [36]:

$$
\tau_{\mathrm{B}}^{i}=\frac{12 \pi \eta r_{i h}^{3}}{k_{\mathrm{B}} T},
$$

where $r_{i h}$ is hydrodynamic radius and $\eta$ is the dynamic viscosity of the carrier liquid. We define an effective relaxation time [36], in the form

$$
\frac{1}{\tau_{\mathrm{eff}}^{i}}=\frac{1}{\tau_{\mathrm{N}}^{i}}+\frac{1}{\tau_{\mathrm{B}}^{i}} \text { or } \tau_{\mathrm{eff}}^{i}=\frac{\tau_{\mathrm{N}}^{i} \tau_{\mathrm{B}}^{i}}{\tau_{\mathrm{N}}^{i}+\tau_{\mathrm{B}}^{i}}
$$

\section{Impact of methods in calculating the magnetic dipolar interaction energy on the relaxation time of a magnetic nanoparticle systems}

In order to assess the impact of methods in calculating the magnetic dipolar interaction energy on the relaxation time of a magnetic nanoparticle systems we develop a numerical calculation. The nanoparticles have been located within a base-centred cubic lattice. Among all the possible discrete positions, the particles have been centred in randomly selected suitable locations (e.g., Fig. 2 for $10^{3}$ nanoparticles). The simulation box has been dimensioned according to the volume fraction of nanoparticles. We assumed periodic boundary conditions on all the sides of the simulation box.

\subsection{The simulation parameters}

We consider a system composed by uncoated ironoxide spherical magnetic nanoparticles, with the saturation magnetization $M_{s}=4.46 \times 10^{5} \mathrm{~A} / \mathrm{m}$, average diameter $d_{m}=10 \mathrm{~nm}$. The diameters of the particles have a 


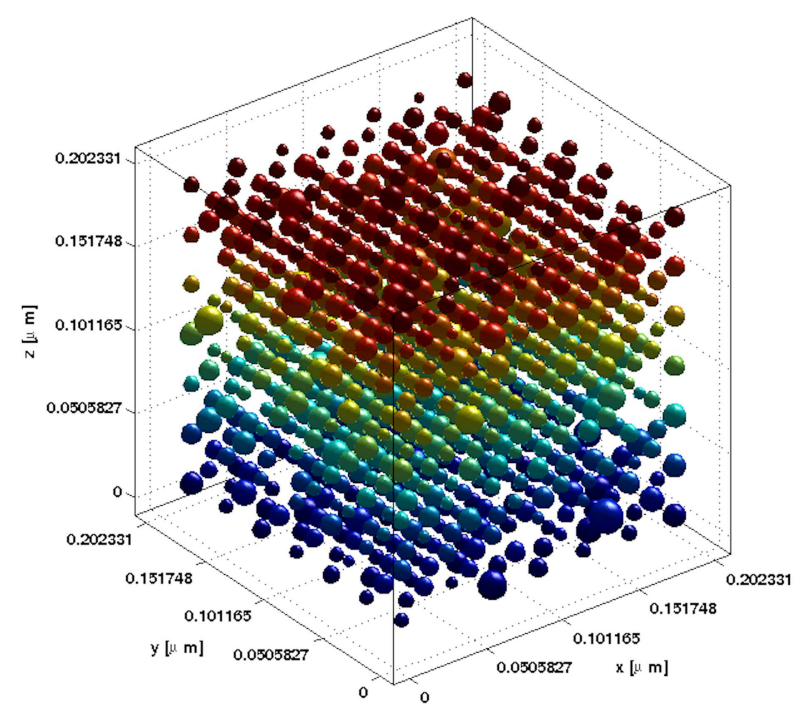

Fig. 2. 3D random distribution for $10^{3}$ nanoparticles at the volume fraction $f=0.1$, average diameter $10 \mathrm{~nm}$ and lognormal distribution with 0.198 standard deviation.

lognormal distribution motivated by experimental measurements [37]. Standard deviation for diameter is $0.2 d_{m}$ in our calculations. The most common type of magnetic nanoparticle anisotropy are: magnetocrystalline anisotropy or magnetic anisotropy, surface anisotropy, and shape anisotropy [17]. All these anisotropy have influence on the magnetic properties in different ways [38]. The total magnetic anisotropy of a spherical nanoparticle, characterized by that effective anisotropy constant, includes the contributions of surface and core of nanoparticle is given by the phenomenological expression [17]:

$$
K_{\text {eff }}=K_{v}+6 \frac{K_{s}}{d},
$$

where $K_{v}$ is the magnetic anisotropy of the core (uniaxial for spherical nanoparticle), $K_{s}$ is the surface anisotropy of the nanoparticle and $d$ is the diameter of the nanoparticle. According to Eq. (39), we considered a lognormal distribution of effective anisotropy constants with average $\mu_{K_{\text {eff }}}=10^{4} \mathrm{~J} / \mathrm{m}^{3}$ and standard deviation $0.1 K_{\text {eff }}$. The intensity of the external magnetic field applied is $15 \mathrm{kA} / \mathrm{m}$, which is typical for the magnetic hyperthermia applications. The analysed systems contain $N=10^{3}$ and $N=10^{4}$ nanoparticles, in a base fluid with the dynamic viscosity of $6 \times 10^{-3} \mathrm{~Pa} \mathrm{~s}$, at the temperature $T=298 \mathrm{~K}$. The nanoparticles' volume fraction varies between 0.02 and 0.1 .

\subsection{Multi-criteria analysis of the pseudorandom number generators}

For accurate simulations, we also studied pseudorandom generators, according to the super decision model (Fig. 3, based on the multi-criteria analytical hierarchy process (AHP) method [39, 40].

We simulated an in-study case of 1000 particles by direct summation, taking into account the average Néel relaxation time $\left(\bar{\tau}_{\mathrm{N}}\right)$ in groups of 50 data, with 3 types of pseudorandom generators, presented in Table I, for various values of the seed [41]. The execution times have been recorded and are displayed in Table II.

TABLE I

Utilitary parameters of pseudorandom generator algorithms: multiple stream and substream support (A) and approximate period in full precision (B).

\begin{tabular}{l|c|c}
\hline \hline \multicolumn{1}{c|}{ generator } & $\mathrm{A}$ & $\mathrm{B}$ \\
\hline Mersenne Twister (MT) & no & $2^{19936}-1$ \\
\hline $\begin{array}{l}\text { Combined multiple } \\
\text { recursive generator (MRG) }\end{array}$ & yes & $2^{127}$ \\
\hline $\begin{array}{l}\text { Multiplicative lagged } \\
\text { Fibonacci generator (MLFG) }\end{array}$ & yes & $2^{124}$
\end{tabular}

TABLE II

Value (top) and execution time $\bar{\tau}_{\mathrm{N}}$ (bottom) for simulated data.

\begin{tabular}{c|c|c|c}
\hline \hline \multirow{2}{*}{ seed } & \multicolumn{3}{|c}{ Generator } \\
\cline { 2 - 4 } & MT & MRG & MLFG \\
\hline \multirow{2}{*}{10} & $8.601568 \times 10^{-10}$ & $1.064920 \times 10^{-9}$ & $8.725124 \times 10^{-10}$ \\
& 2.28874576 & 2.32073514 & 2.3254319 \\
100 & $8.501283 \times 10^{-10}$ & $9.094906 \times 10^{-10}$ & $8.640541 \times 10^{-10}$ \\
& 2.29193154 & 2.29078382 & 2.29079342 \\
1000 & $8.620694 \times 10^{-10}$ & $8.611658 \times 10^{-10}$ & $8.815158 \times 10^{-10}$ \\
& 2.28615483 & 2.32560564 & 2.35758171
\end{tabular}

The alternatives representing the 3 types of random number generators can be ranked based on the obtained priorities. In fact, the three levels of the model contain nodes that define the elements of the problem, i.e. the purpose, the criteria, and possible alternatives, and are inter-connected in top-down direction. The level that includes the criteria is selected with equal importance weight, and the alternatives are assessed according to preferences in relation to the criteria. Once this hierarchical structure is defined, the elements on each level are analysed in a procedure for prioritizing, settling their relative importance on each level.

Two types of criteria are established in this analysis, i.e.:

- the numerical criteria (Time1, Time2 and Time3), representing the execution times resulted from the measurements made for each seed;

- the non-numerical criterion (quality), representing the approximate period in full precision.

Based on the AHP methodology, the determination of relative weights of criteria is made by comparing the alternatives two by two, relative to the objective found at the upper level of the hierarchy. The pairwise comparisons are performed by assessing the generators based on a scale from 1 to 9 , as shown in Table III and representing the degree of importance.

The result of quantification for these criteria (Priorities versus the Quality parameter) shown that the Mersenne 


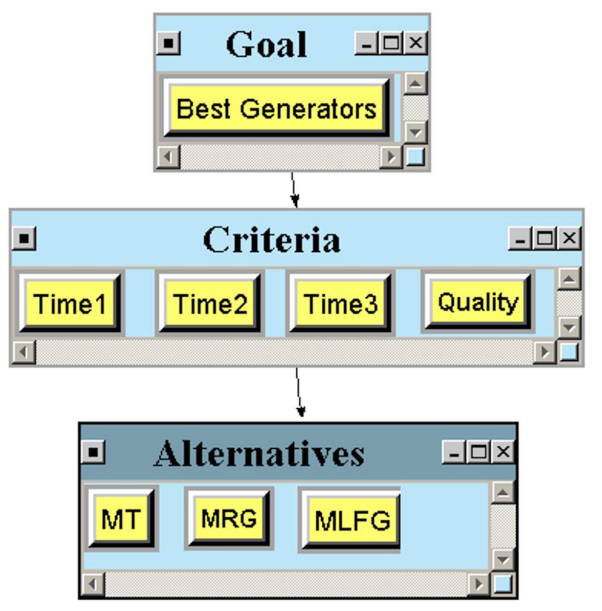

Fig. 3. The super decision model "Types of generators".

TABLE III

The scale of relative preferences [40].

\begin{tabular}{|c|c|c|}
\hline \multicolumn{2}{|c|}{ importance } & \multirow{2}{*}{ explanations } \\
\hline level & grade & \\
\hline 1 & equal & $\begin{array}{l}\text { two elements contribute equally to } \\
\text { the objective }\end{array}$ \\
\hline 3 & less & $\begin{array}{l}\text { the experience and judgment are } \\
\text { slightly favouring one over the other } \\
\text { element }\end{array}$ \\
\hline 5 & middle & $\begin{array}{l}\text { the experience and judgment } \\
\text { are favouring one over the other } \\
\text { element }\end{array}$ \\
\hline 7 & high & $\begin{array}{l}\text { An element is strongly favoured and } \\
\text { its domination is demonstrated in } \\
\text { practice }\end{array}$ \\
\hline 9 & extreme & $\begin{array}{l}\text { the element is clearly favoured over } \\
\text { the other one }\end{array}$ \\
\hline $2,4,6,8$ & interm. & \\
\hline
\end{tabular}

Twister generator is firstly ranked and, then, it should be preferred than the MRG or MLFG in our simulations.

\section{Results and discussions}

After the selection of the suitable algorithm for generating random numbers, our first study involves the simulation of the average Néel relaxation time $\bar{\tau}_{\mathrm{N}}$ and the effective average relaxation time $\bar{\tau}_{\text {eff }}$ at volume fractions ranging between 0.02 and 0.1 . We exploited the four presented methods for calculating the dipolar magnetic interaction energy and compare with analytical (or "brute force") method, i.e. the calculation of local magnetic field on the nanoparticle.

We applied the Approximation 1, 2, 3, Ewald summation method and analytical (or "brute force") method with randomly oriented magnetic moments. The results are proposed in Fig. 4. It is possible to note how the applied approximation method influences the results obtained for $\bar{\tau}_{\mathrm{N}}$ and $\bar{\tau}_{\text {eff }}$. It can be also seen that the "Approximation 1" and "Approximation 2" provide about the same results.
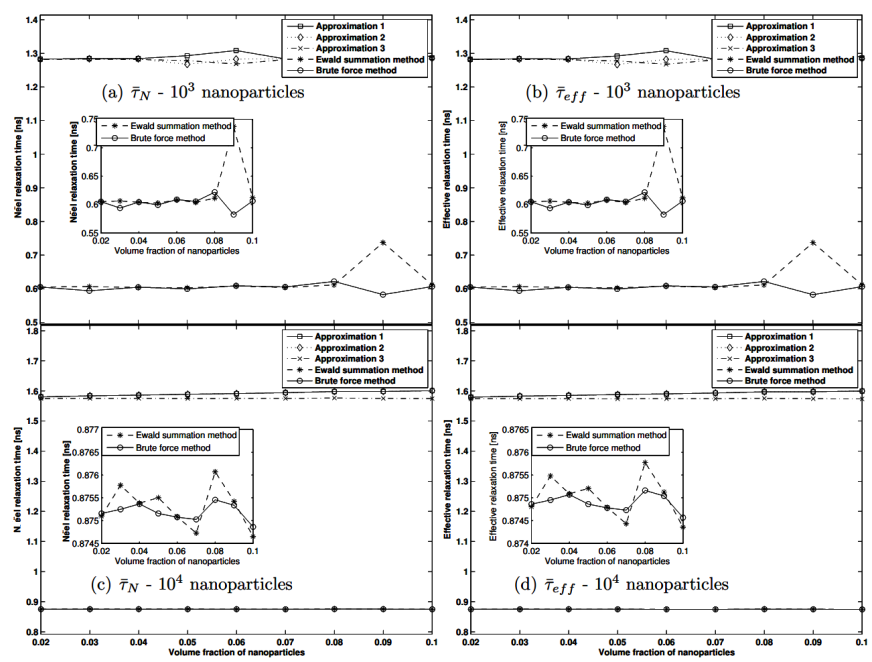

Fig. 4. Average Néel and effective relaxation times versus the volume fraction of colloid nanoparticles. The nested graph is a zoom on Ewald and "brute force" methods only. (a) $-\bar{\tau}_{N}=10^{3}$ nanoparticles, (b) $\bar{\tau}_{\text {eff }}=10^{3}$ nanoparticles, (c) $-\bar{\tau}_{N}=10^{4}$ nanoparticles, (d) $-\bar{\tau}_{\text {eff }}=10^{4}$ nanoparticles.

Note that, if the calculation is performed with the Ewald summation method and "brute force" method, $\bar{\tau}_{N}$ (or $\bar{\tau}_{\text {eff }}$ ) decreases slightly with increasing volume fraction. We noted that the results obtained by Ewald summation method and by "brute force" methods are very similar. The slight decrease of relaxation time with the increase of volume fraction has been experimentally observed in dilute systems [8, 42, 24], when the Mössbauer spectroscopy measurements on weakly interacting $\gamma-\mathrm{Fe}_{2} \mathrm{O}_{3}$ nanoparticles showed a decrease of the relaxation time with the increase of interparticle interaction. In studying the specific absorption rate (SAR) of a maghemite-based ferrofluid, Urtizberea et al. [43] measured $3 \mathrm{kA} / \mathrm{m}$ and $10^{9} \mathrm{kHz}$ at $315 \mathrm{~K}$. The magnetic characterization of three different concentrations of this ferrofluid revealed a decrement of initial susceptibility values and Néel relaxation times with the increase of concentration. By using the four approximation methods presented in this paper, we simulated the average dipolar magnetic energy and the average energy barriers $E_{\mathrm{b} 12}, E_{\mathrm{b} 21}$, including the average effective energy barrier $E_{\mathrm{b} m}=\left(E_{\mathrm{b} 12}+E_{\mathrm{b} 21}\right) / 2$ (Figs. 5, 6). Regarding the dipolar magnetic energy, since the method "Approximation 1" and "Approximation 2" provide the highest value for the dipolar magnetic energy, since they are working with aligned and parallel magnetic moments. The DormannBessais-Fiorani method ("Approximation 3") offers lower values, because it only considers the component of the local dipolar field along the direction of external magnetic field. Finally, the Ewald summation method gives lower dipolar magnetic energies, because we applied it in more general conditions, i.e., without imposing a preferred orientation of the easy axes and magnetic moments. Please 
note that the results obtained for dipolar magnetic energies by the Ewald summation method and by "brute force" method are very similar.

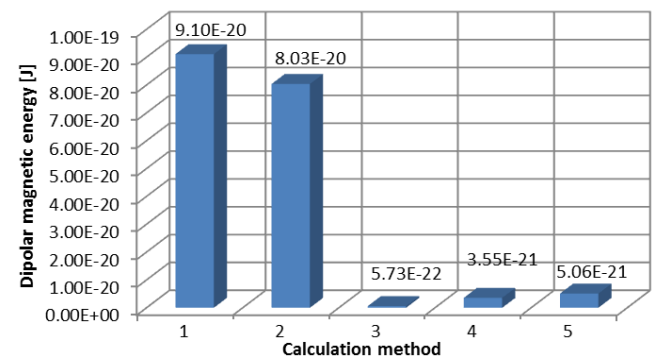

Fig. 5. The average dipolar magnetic energy for a system with $10^{3}$ particles with the volume fraction 0.1 , calculated by: "Approximation 1" (1st series), "Approximation 2" (2nd series), "Approximation 3" (3d series), Ewald summation method (4th series), analytical (or "brute force") method (5th series).

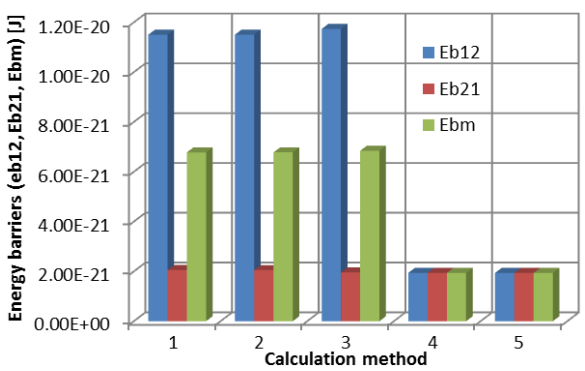

Fig. 6. Energy barriers for a system with $10^{3}$ particles with the volume fraction 0.1 , calculated by: "Approximation 1" (1st series), "Approximation 2" (2nd series), "Approximation 3" (3d series), Ewald summation method (4th series), analytical (or "brute force") method (5th series).

Analysing the average energy barriers, we can see that there are no significant differences among the simulations performed by using the first three approximation methods. Instead, when working with the Ewald summation method in terms of random distribution of anisotropy easy axes, we used the Pfeiffer approximation for the energy barrier (Eq. (35)) [33]. Usage of "Approximation 1 " and "Approximation 2" in case of random distribution of anisotropy easy axes is not suitable. There are many papers in the literature, especially related to magnetic hyperthermia, which not consider dipolar magnetic interactions or consider alignment of magnetic moments along the direction of the external magnetic field even in weak fields [44-46]. Actually, the oriented state of the magnetic nanoparticles changes dramatically depending on the size and shape of the nanoparticles, the viscosity of their surroundings, and the alternating magnetic field conditions. Also, recent researches show that the influence of magnetic dipolar interactions cannot be neglected in the case of low concentrations of nanoparti- cles [17]. The following Table IV shows the runtime for running the various methods. The simulations have been carried out with a $2.13 \mathrm{GHz}$ Intel ${ }^{\circledR}$ Core $^{T M}$ P7450 processor (1066 MHz FSB, 3 MB L2 cache) with 4 GB DDR3 RAM.

TABLE IV

Runtime for different approximation methods and particle numbers.

\begin{tabular}{c|c|c|c}
\hline \hline \multirow{2}{*}{ type } & \multicolumn{3}{|c}{ runtime $[\mathrm{s}]$} \\
\cline { 2 - 4 } & 1000 & 5000 & 10000 \\
\hline App. 1 & 1.401118 & 16.470398 & 64.573375 \\
App. 2 & 2.896265 & 39.216689 & 142.791277 \\
App. 3 & 1.388862 & 15.912926 & 54.220290 \\
Ewald & 2.536140 & 22.494049 & 81.969832 \\
direct & 2.286039 & 27.447619 & 96.228571
\end{tabular}

\section{Conclusions}

Within this paper, we dealt with the mostly used approximations methods for the calculation of long range magnetic dipolar interaction energy. Our aim was to study how these methods influence the Néel relaxation time and the effective relaxation time for a nanofluid.

Usually, when a system includes a relatively low number of nanoparticles, analytical, i.e., "brute force" method is suitable to be implemented. But, when the number of nanoparticles increases dramatically, the time of calculation of "brute force" method seriously rises since its computational complexity equals to $O\left(n^{2}\right)$, with $n$ the number of nanoparticles. Therefore, a system with a huge number of nanoparticles (e.g. $10^{7}$ and higher) is difficult to be managed by a single computer, and it needs dedicated resources of calculation to be solved. This is the reason to exploit approximation methods, so that the computational complexity (and thus the time of calculation) can be reduced. Therefore, a comparison with analytical results is useful to establish which kind of approximation method should be exploited, and in which simulation conditions. That is why we restrict the comparison to systems having $10^{3}$ or $10^{5}$ nanoparticles: we aim to establish the usefulness of approximation methods with numbers of nanoparticles which can be handled by usual computing systems, in order to be furtherly exploited for greater numbers of nanoparticles.

Our studies show that the approximation methods affect pretty much the Néel relaxation time, the effective relaxation time, and other quantities of a nanoparticlebased colloidal system. Therefore, a suitable choice of the approximation method is required, in order to have robust results. The choice of the approximation method depends on the condition of the in-study nanofluid, especially if it is saturated or not when an external magnetic field is applied. If it is highly saturated, we can work with the "Approximation 1" (Table IV).

If the degree of magnetic saturation is lower but magnetic anisotropy axis of nanoparticles are aligned, we can try the "Approximation 3" (Table IV). The "Approximation 2" (Table IV) is instead efficient at small particle 
number but shows inefficiency for high number of particles. In order to have a realistic simulated system, we have to accurately assess the approximation conditions when using the methods "Approximation 1", "Approximation 2" or "Approximation 3", since conditions can affect the results. Moreover, the problem of time efficiency of the applied algorithm arises. When the system contains many nanoparticles, the method "Approximation 2" is not computationally efficient in time, being more time consuming than direct summation method (Table IV). It is possible to note how the results obtained for dipolar magnetic energies by the Ewald summation method and by "brute force" method are very similar. The Ewald summation allows for application of short cut-off distances without the loss of accuracy. In our work, we stated to cut-off parameters in real and Fourier space, as well as the optimal $\alpha$ value, according to the method proposed by $[47,48]$. The Ewald summation method has a more constant time efficiency with the increase of the number of nanoparticles. It is due to its intrinsic formulation, since it is a special case of the Poisson summation formula, replacing the summation of interaction energies in real space with an equivalent summation in Fourier space. Therefore, we have a more rapid convergence of the energy than the direct summation. This means that the method has high accuracy and reasonable speed when computing long-range interactions. Moreover, the use of Ewald summation method does not require the knowledge of information about the degree of magnetic saturation, being a general method for the evaluation of magnetic dipole-dipole interactions in the presence of periodic boundary conditions. It also offers a computational efficiency, because the conditionally convergent dipole-dipole interaction energy sum is split into rapidly convergent real-space and Fourier-space series, which are truncated in order to evaluate the energy to a desired accuracy.

\section{References}

[1] V. Sreeja, P.A. Joy, Int. J. Nanotechnol. 8, 907 (2011).

[2] Q.A. Pankhurst, J. Connolly, S.K. Jones, J. Dobson, J. Phys. D Appl. Phys. 36, R167 (2003).

[3] T. Neuberger, B. Schopf, H. Hofmann, M. Hofmann, B. von Rechenberg, J. Magn. Magn. Mater. 293, 483 (2005).

[4] D.V. Berkov, L.Yu. Iskakova, A.Yu. Zubarev, Phys. Rev. E 79, 021407 (2009).

[5] M. Panoiu, C. Panoiu, I. Sora, M. Osaci, Int. J. Modell. Identific. Control 2, 250 (2007).

[6] M. Osaci, M. Panoiu, I. Mascalagiu, C. Panoiu, Computat. Mater. Sci. 27, 523 (2003).

[7] D.A. Bistrian, Comput. Math. Appl. 68, 197 (2014).

[8] D.A. Bistrian, Appl. Math. Modell. 37, 3993 (2013).

[9] M.F. Hansen, S. Morup, J. Magn. Magn. Mater. 184, 262 (1998).

[10] D. Serantes, D. Baldomir, Open Surf. Sci. J. 4, 71 (2012).
[11] S. Ruta, R. Chantrell, O. Hovorka, Sci. Rep. 5, 9090 (2015).

[12] O.A. Chubykalo-Fesenko, R.W. Chantrell, J. Magn. Magn. Mater. 343, 189 (2004).

[13] R. Prozorov, Y. Yeshurun, T. Prozorov, A. Gedanken, Phys. Rev. B 59, 6956 (1999).

[14] M. Osaci, J. Magn. Magn. Mater. 343, 189 (2013).

[15] M. Osaci, M. Panoiu, T. Heput, I. Mascalagiu, 10.1016/j.apm.2005.11.013Appl. Math. Modell. 30, 545 (2006).

[16] M. Osaci, Proc. Roman. Acad. Ser. A Math. Phys. Techn. Sci. Inform. Sci. 11, 137 (2010).

[17] I.M. Obaidat, B. Issa, Y. Haik, Nanomaterials 5, 63 (2015).

[18] B. Huke, M. Lücke, Rep. Prog. Phys. 67, 1731 (2004).

[19] C. Haase, U. Nowak, Phys. Rev. B 85, 045435 (2012).

[20] S. Morup, M.F. Hansen, C. Frandsen, Beilstein J. Nanotechnol. 1, 182 (2010).

[21] A.C. Silva, T.R. Oliveira, J.B. Mamani, Int. J. Nanomed. 6, 591 (2011).

[22] I.S. Jacobs, C.P. Bean, Phys Rev. 100, 1060 (1955).

[23] M. Jeun, S. Bae, A. Tomitaka, Ki Ho Park, S.H. Paek, K.W. Chung, Appl. Phys. Lett. 95, 082501 (2009).

[24] S. Morup, E. Tronc, Phys. Rev. Lett. 72, 3278 (1994).

[25] J.L. Dormann, L. Bessais, D. Fiorani, J. Phys. C Solid State Phys. 21, 2015 (1988).

[26] Z. Wang, C. Holm, J. Chem. Phys. 115, 6277 (2001).

[27] J.W. Perram, S.W. de Leeuw, E.R. Smith, Proc. $R$. Soc. Lond. A 373, 27 (1980).

[28] T. Darden, D. York, L. Pedersen, J. Chem. Phys. 98, 10089 (1993).

[29] A. Lherbier, S. Roche, O.A. Restrepo, Y.-M. Niquet, A. Delcorteand, J.-C. Charlier, Nano Res. 6, 326 (2013).

[30] H. Wang, S. Michielssens, S.L.C. Moors, A. Ceulemans, Nano Res. 2, 945 (2009).

[31] M. Lanza, Y. Wang, T. Gao, A. Bayerl, M. Porti, M. Nafria, Y. Zhou, G. Jing, Y. Zhang, Z. Liu, D. Yu, H. Duan, Nano Res. 6, 485 (2013).

[32] W.T. Coffey, D.S.F. Crothers, J.L. Dormann, L.J. Geoghegan, P.Yu. Kalmykov, J.T. Waldron, A.W. Wickstead, Phys. Rev. B 145, L263 (1995).

[33] H. Pfeiffer, Phys. Status Solidi A 118, 295 (1990).

[34] L. Lopez-Diaz, L. Torres, E. Moro, Phys. Rev. B 65, 224406 (2002).

[35] F. Reif, Fundamentals of Statistical and Thermal Physics, McGraw-Hill, New York 1965.

[36] V. Singh, V. Banerjee, M. Sharma, J. Phys. D Appl. Phys. 42, 245006 (2009).

[37] J.B. Mamani, L.F. Gamarra, G.E. de Souza Brito, Mater. Res. 17, 542 (2014).

[38] M. Osaci, Proc. Roman. Acad. Ser. A Math. Phys. Techn. Sci. Inform. Sci. 8, 37 (2007).

[39] E. Triantaphyllou, S.H. Mann, Int. J. Industr. Eng. Appl. Pract. 2, 35 (1995). 
[40] L.T. Saaty, Manag. Sci. 36, 259 (1990).

[41] M. Osaci, Acta Techn. Corvininesis Bull. Eng. 7, 103 (2014).

[42] P. Prené, E. Tronc, J.P. Jolivet, J. Livage, R. Cherkaoui, M. Nogués, J.L. Dormann, D. Fiorani, IEEE Trans. Magn. 29, 2658 (1993).

[43] A. Urtizberea, E. Natividad, A.A. Miguel Castro, A. Mediano, J. Phys. Chem. C 114, 4916 (2010).

[44] C.L. Ondeck, A.H. Habib, P. Ohodnicki, K. Miller, C.A. Sawyer, P. Chaudhary, M.E. McHenry, J. Appl. Phys. 105, 07B324 (2009).
[45] S. Purushotham, R.V. Ramanujan, J. Appl. Phys. 107, 114701 (2010).

[46] Xuman Wang, Hongchen Gu, Zhengqiang Yang, $J$. Magn. Magn. Mater. 293, 334 (2005).

[47] J. Kolafa, J.W. Perram, Mol. Simul. 9, 351 (1992).

[48] Z. Wang, C. Holm, J. Chem. Phys. 115, 6351 (2001). 